\title{
Avaliação de seis variedades de uvas sem sementes no Submédio São Francisco(1)
}

\author{
Patrícia Coelho de Souza Leão(2) e Fernando Mendes Pereira ${ }^{(3)}$
}

\begin{abstract}
Resumo - Este trabalho teve como objetivo avaliar aspectos relacionados ao desenvolvimento vegetativo e produtivo das plantas, às características físicas e à composição química dos frutos de seis variedades de uvas sem sementes nas condições do Submédio São Francisco. Os dados foram registrados durante os anos de 1997 e 1998. As variedades utilizadas foram enxertadas sobre o porta-enxerto IAC 572 ('Jales'). O delineamento experimental foi inteiramente casualizado, com parcelas subdivididas nos ciclos de produção. Houve diferenças significativas na resposta da maioria das variedades entre as épocas de poda. O peso médio dos cachos variou entre 164,8 g na variedade Marroo Seedless, e 203,5 g na 'Beauty Seedless'. O diâmetro médio das bagas foi superior a $15,7 \mathrm{~mm}$ em todas as variedades. Foram obtidos teores de sólidos solúveis compreendidos entre $14,05^{\circ}$ Brix na variedade Canner e $19,6^{\circ}$ Brix na 'Vênus', enquanto a acidez total titulável foi inferior a $0,91 \mathrm{~g}$ de ácido tartárico/100 $\mathrm{mL}$ de suco, resultando em adequadas relações SST/ATT, que variaram entre 19,05 na variedade Beauty Seedless a 28,57 na 'Vênus'. As variedades Vênus e Marroo Seedless foram as mais produtivas, com produtividades anuais de $24 \mathrm{t} / \mathrm{ha}$ e $20 \mathrm{t} / \mathrm{ha}$, respectivamente.
\end{abstract}

Termos para indexação: Vitis vinifera, videira, frutas, etapas de desenvolvimento da planta, rendimento.

Evaluation of six seedless grape varieties under the conditions of São Francisco river's valley

\begin{abstract}
This work aimed to study aspects related to vegetative and productive development of plants and characteristics and chemical components of bunches of six seedless grape varieties at environmental conditions of São Francisco river's valley, Northeastern Brazil. The experiment was carried out during 1997 and 1998. The varieties tested were grafted on IAC 572 ('Jales') rootstock. The experimental model utilized was totally randomized, and with parcels subdivided into different production cycles. The results showed significative differences among varieties at different pruning times. The average weight of bunches varied from $164.8 \mathrm{~g}$ for 'Marroo Seedless' and $203.5 \mathrm{~g}$ for 'Beauty Seedless'. The average diameter of berries was superior to $15.7 \mathrm{~mm}$ for all of the varieties. The total soluble solids varied from $14.05^{\circ}$ Brix in 'Canner' to $19.6^{\circ}$ Brix in 'Venus'. The total titratable acidity (ATT) was lower than $0.91 \mathrm{~g}$ tartaric acid/100 $\mathrm{mL}$ of juice. The ratio SST/ATT varied from 19.05 in 'Beauty Seedless' to 28.57 in 'Vênus'. 'Vênus' and 'Marroo Seedless' were the most productive varieties, with the average annual production of 24 ton/ha and 20 ton/ha, respectively.
\end{abstract}

Index terms: Vitis vinifera, grapevines, fruits, plant developmental stages, yield.

(1) Aceito para publicação em 8 de dezembro de 2000 .

Extraído da dissertação de mestrado, apresentada pelo primeiro autor à Universidade Estadual Paulista (UNESP), Jaboticabal, SP.

(2) Embrapa-Centro de Pesquisa Agropecuária do Trópico SemiÁrido, Caixa Postal 23, CEP 56300-000 Petrolina, PE. E-mail: patricia@cpatsa.embrapa.br

(3) UNESP, FCAV, Dep. de Horticultura, Via de acesso Prof Paulo Donatto Castellane, km 5, CEP 14870-000 Jaboticabal, SP. E-mail: nlynn@fcav.unesp.br

\section{Introdução}

O Vale do Rio São Francisco, na região Nordeste do Brasil, apresenta uma vocação natural para a produção de uvas finas de mesa. A variedade Itália e outras com sementes representam quase $100 \%$ da área cultivada com uvas de mesa nesta região.

Nesta última década, observou-se um crescente interesse dos viticultores pela produção de uvas sem 
sementes, como conseqüência dos seguintes aspectos principais: seguir as tendências de consumo do mercado internacional; buscar uma melhoria de qualidade para atender às exigências dos importadores e oferecer novas alternativas de variedades no mercado interno, especialmente em um contexto de mercado globalizado, com a presença cada vez mais forte de variedades de uva sem sementes nos supermercados brasileiros.

Atualmente, o principal fator de ordem técnica limitante à expansão da viticultura no Nordeste brasileiro está relacionado à concentração da produção na variedade Itália, e entre as principais demandas tecnológicas, destaca-se a obtenção de variedades de uvas sem sementes adaptadas às condições ambientais do Vale do Rio São Francisco (Silva et al., 1998).

Este trabalho teve como objetivo avaliar aspectos relacionados ao desenvolvimento vegetativo e produtivo das plantas e às características e à composição química dos frutos de seis variedades de uvas sem sementes, durante cinco ciclos de produção, a fim de se selecionar aquelas melhores adaptadas às condições tropicais semi-áridas.

\section{Material e Métodos}

O experimento foi conduzido no Campo Experimental de Bebedouro, pertencente à Embrapa-Centro de Pesquisa Agropecuária do Trópico Semi-Árido, localizado no Município de Petrolina, PE, latitude $9^{\circ} 9^{\prime}$ Sul, longitude $40^{\circ} 22^{\prime}$ Oeste, e altitude média de $365,5 \mathrm{~m}$. Segundo a classificação de Köppen, o clima da região pode ser classificado como tipo Bswh, que corresponde a uma região semiárida muito quente, com temperatura média anual de $26,4^{\circ} \mathrm{C}$, média das mínimas de $20,6^{\circ} \mathrm{C}$, e média das máximas $31,7^{\circ} \mathrm{C}$. Os dados climatológicos referentes ao período de realização do trabalho são apresentados na Figura 1.

O vinhedo no qual se realizou o experimento foi formado por uma coleção de variedades de uva sem sementes, instalado em setembro de 1994, utilizando-se como portaenxerto a cultivar IAC 572 'Jales'. O sistema de condução empregado foi a latada, com espaçamento de 4,00 × 2,00 m (1.250 plantas/ha), e sistema de irrigação localizada, do tipo gotejamento. O período considerado no estudo correspondeu aos anos de 1997 e 1998, sendo efetuadas avaliações de cinco ciclos de produção. As datas de poda foram 14/1/1997, 16/6/1997, 13/11/1997, 25/3/1998 e $10 / 8 / 1998$
Foram utilizadas treze plantas para avaliação da produção e desenvolvimento vegetativo. Entre as treze plantas foram identificadas três, marcadas com etiquetas e consideradas como amostra para caracterização dos cachos, bagas e composição química dos frutos. As mesmas plantas foram avaliadas em todos os ciclos de produção considerados neste estudo. Com os dados obtidos determinou-se a relação sólidos solúveis totais/acidez total titulável (ratio).

O delineamento experimental utilizado foi inteiramente casualizado, em esquema de parcelas subdivididas, tendo como parcelas as variedades, e como subparcelas, os ciclos de produção.
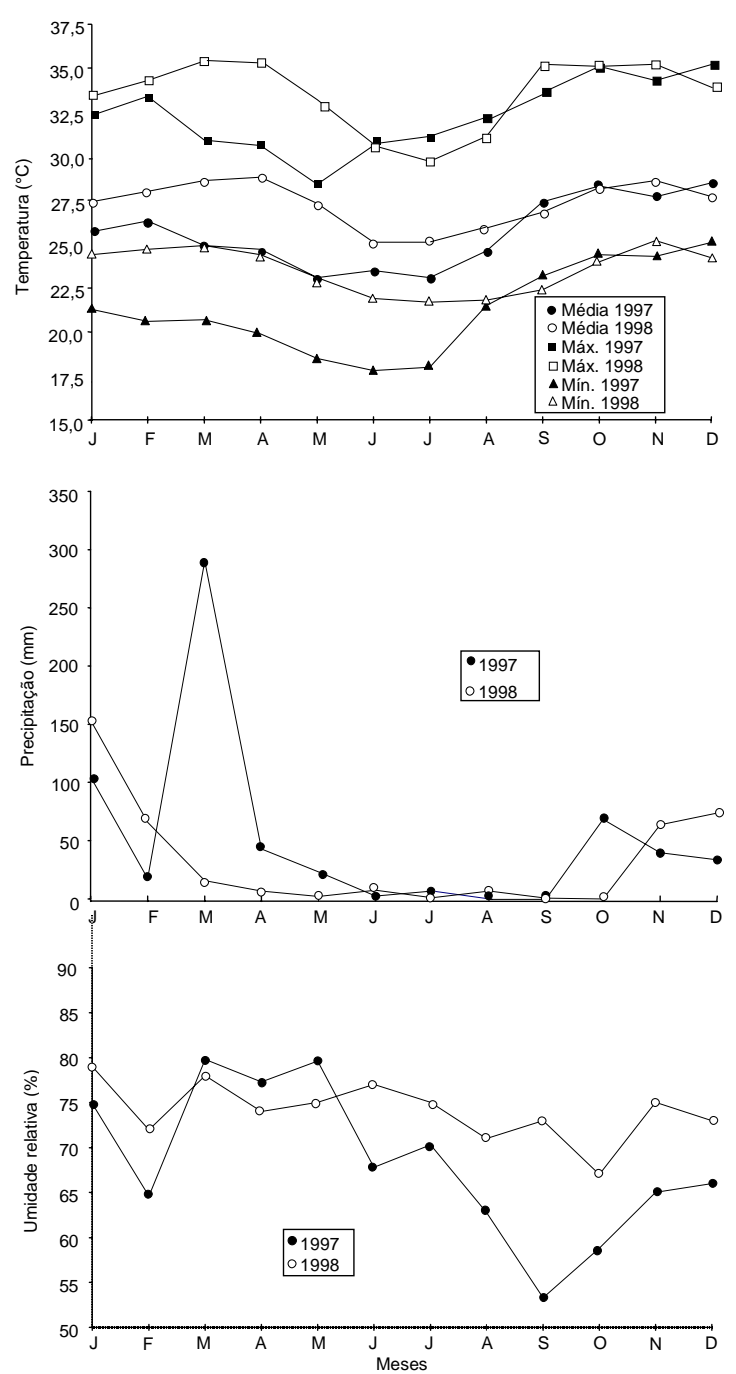

Figura 1. Médias mensais de temperatura, precipitação e umidade relativa durante os anos de 1997 e 1998, Petrolina, PE. 
Os dados obtidos foram analisados estatisticamente, estabelecendo-se a comparação das médias pelo teste de Tukey a $5 \%$ de probabilidade.

\section{Resultados e Discussão}

O peso médio dos cachos apresentou diferenças significativas entre os ciclos de produção apenas nas variedades Thompson Seedless e Canner (Tabela 1). Entretanto, pode-se observar uma tendência comum em todas as variedades em que os cachos com maiores pesos obtidos no segundo ciclo (poda $16 / 6 / 97)$ decresceram durante o terceiro e quarto ciclos (podas 13/11/97 e 25/3/98) e voltaram a aumentar no quinto ciclo de produção.

No quarto ciclo de produção, o comprimento dos cachos foi menor do que no quinto ciclo nas variedades Arizul, Beauty Seedless e Canner, e em relação à largura dos cachos nas variedades Beauty Seedless e Canner. Os resultados de tamanho de cachos, apesar de atenderem os padrões comerciais, foram infe- riores aos encontrados na literatura em outras regiões, nestas variedades (Calò et al., 1989; Ferri, 1994; Schuck; 1994), o que evidencia que as condições climáticas e o sistema de produção do Vale do Rio São Francisco não permitiram plena expressão das características das seis variedades estudadas.

Quanto ao tamanho das bagas, as variedades apresentaram diferenças significativas entre os ciclos (Tabela 2). No primeiro e no quinto ciclos ocorreram bagas de maior e menor diâmetro respectivamente nas variedades Vênus e Marroo Seedless. O comprimento médio das bagas variou entre $16,69 \mathrm{~mm}$ na variedade Vênus e $21 \mathrm{~mm}$ na variedade Canner. Por outro lado, o diâmetro médio das bagas ficou compreendido entre $15,7 \mathrm{~mm}$ na variedade Beauty Seedless e 18,26 mm na variedade Marroo Seedless. 'Marroo Seedless' e 'Vênus' destacaramse com as maiores médias em volume e peso das bagas em quase todos os ciclos, enquanto 'Beauty Seedless' apresentou o menor volume e peso das bagas entre as variedades no primeiro, segundo e quinto ciclos de produção.

Tabela 1. Valores médios das variáveis relacionadas a características dos cachos de seis variedades de uvas sem sementes durante cinco ciclos de produção. Petrolina, PE, 1997-1998 ${ }^{(1)}$.

\begin{tabular}{|c|c|c|c|c|c|c|c|}
\hline \multirow[t]{2}{*}{ Variável } & \multirow[t]{2}{*}{ Ciclo } & \multicolumn{6}{|c|}{ Variedade } \\
\hline & & Vênus & Arizul & Beauty Seedless & $\begin{array}{l}\text { Thompson } \\
\text { Seedless }\end{array}$ & $\begin{array}{l}\text { Marroo } \\
\text { Seedless }\end{array}$ & Canner \\
\hline \multirow[t]{6}{*}{ Peso de cachos (g) } & $1^{\mathrm{o}}$ & $135,00 \mathrm{a}$ & $251,67 \mathrm{a}$ & $174,00 \mathrm{a}$ & $134,33 b$ & $152,00 \mathrm{a}$ & $145,67 b$ \\
\hline & $2^{\underline{o}}$ & $212,67 \mathrm{a}$ & $292,00 \mathrm{a}$ & $232,00 \mathrm{a}$ & $308,33 a$ & $182,33 a$ & $240,00 \mathrm{ab}$ \\
\hline & $3^{-}$ & $154,00 \mathrm{a}$ & $179,67 \mathrm{a}$ & $165,33 \mathrm{a}$ & $94,67 b$ & $147,00 \mathrm{a}$ & $131,67 b$ \\
\hline & $4^{\mathrm{o}}$ & $133,67 \mathrm{a}$ & $134,67 \mathrm{a}$ & $163,33 \mathrm{a}$ & $138,33 b$ & $168,67 \mathrm{a}$ & $132,33 b$ \\
\hline & $5^{\circ}$ & $219,33 a$ & $199,33 a$ & $283,00 \mathrm{a}$ & $184,00 \mathrm{~b}$ & $174,33 \mathrm{a}$ & $289,00 \mathrm{a}$ \\
\hline & Média & 170,93 & 199,07 & 203,53 & 171,93 & 164,87 & 187,73 \\
\hline \multirow{6}{*}{$\begin{array}{l}\text { Comprimento } \\
\text { de cachos }(\mathrm{cm})\end{array}$} & $1^{o}$ & $13,25 \mathrm{ab}$ & $18,17 \mathrm{a}$ & $15,11 \mathrm{ab}$ & $13,16 \mathrm{~b}$ & $11,80 \mathrm{a}$ & $14,33 a b c$ \\
\hline & $2^{\underline{0}}$ & $16,89 a$ & $16,20 \mathrm{ab}$ & $15,79 \mathrm{ab}$ & $18,44 a$ & $11,84 \mathrm{a}$ & $15,55 \mathrm{ab}$ \\
\hline & $3^{\mathrm{o}}$ & $14,38 \mathrm{ab}$ & $16,19 \mathrm{ab}$ & $15,09 \mathrm{ab}$ & $13,64 b$ & $12,63 \mathrm{a}$ & $12,84 \mathrm{bc}$ \\
\hline & $4^{\circ}$ & $12,50 \mathrm{~b}$ & $13,70 \mathrm{~b}$ & $12,58 \mathrm{~b}$ & $12,09 \mathrm{~b}$ & $11,88 \mathrm{a}$ & $10,72 \mathrm{c}$ \\
\hline & $5^{\circ}$ & $15,34 \mathrm{ab}$ & $18,79 \mathrm{a}$ & $18,43 a$ & $15,28 \mathrm{ab}$ & $14,80 \mathrm{a}$ & $18,12 \mathrm{a}$ \\
\hline & Média & 14,47 & 16,67 & 15,40 & 14,52 & 12,59 & 14,31 \\
\hline \multirow{6}{*}{$\begin{array}{l}\text { Largura de cachos } \\
(\mathrm{cm})\end{array}$} & $1^{\mathrm{O}}$ & $8,35 \mathrm{a}$ & $11,01 \mathrm{ab}$ & $10,36 \mathrm{ab}$ & $7,43 b$ & $8,65 \mathrm{a}$ & $8,62 b$ \\
\hline & $2^{\mathrm{o}}$ & $9,44 a$ & $11,90 \mathrm{a}$ & $10,40 \mathrm{ab}$ & $12,24 \mathrm{a}$ & $9,04 \mathrm{a}$ & $9,70 \mathrm{ab}$ \\
\hline & $3^{\circ}$ & $8,85 a$ & $10,11 \mathrm{ab}$ & $8,95 \mathrm{ab}$ & $7,68 b$ & $8,87 \mathrm{a}$ & $9,07 \mathrm{ab}$ \\
\hline & $4^{\circ}$ & $8,20 \mathrm{a}$ & $7,56 \mathrm{~b}$ & $8,24 b$ & $7,79 \mathrm{~b}$ & $9,01 \mathrm{a}$ & $7,39 \mathrm{~b}$ \\
\hline & $5^{\circ}$ & $10,83 \mathrm{a}$ & $10,75 \mathrm{ab}$ & $12,15 \mathrm{a}$ & $9,13 \mathrm{ab}$ & $11,64 \mathrm{a}$ & $12,36 \mathrm{a}$ \\
\hline & Média & 9,13 & 10,01 & 10,02 & 8,86 & 9,44 & 9,43 \\
\hline
\end{tabular}

${ }^{(1)}$ Médias seguidas pela mesma letra, na coluna, não diferem entre si pelo teste de Tukey $(\mathrm{p}<0,05)$. 
Estas diferenças observadas entre as variedades quanto ao tamanho das bagas eram esperadas, uma vez que esta é uma característica genética e depende do formato da baga de cada variedade. O menor crescimento das bagas, no quinto ciclo de produção, coincidiu com os meses de temperaturas mais elevadas durante a fase de crescimento da baga (Figura 1), e, segundo Hale \& Buttrose (1974), temperaturas elevadas reduzem a duração do estádio I de crescimento, resultando em bagas de menor tamanho. Os resultados obtidos são semelhantes aos encontrados por Camargo et al. (1997) e Leão et al. (1999) em outras variedades de uva sem sementes, na mesma região deste estudo.

O tamanho das bagas obtido em todas as variedades está de acordo com o observado por outros autores em diferentes regiões, nessas mesmas variedades (Moore \& Brown, 1977; Wagner \& Truel, 1988;
Calò et al., 1989; Ferri, 1994; Schuck, 1994; Matheou et al., 1995). As variedades Marroo Seedless e Vênus destacaram-se pelo maior tamanho de suas bagas, exigindo menor quantidade de tratamentos com reguladores de crescimento a fim de atingirem o tamanho desejável das bagas para comercialização.

As épocas de poda exerceram influência na composição química dos frutos, observando-se diferenças significativas entre os ciclos (Tabela 3). Entretanto, foram encontradas respostas diferentes das variedades em relação às condições ambientais predominantes em determinada época de poda ou ciclo de produção. Estas variações eram esperadas, pois de acordo com Calò et al. (1996), a expressão quantitativa dessas características, que são qualitativas, dependem principalmente da interação entre genótipo e clima, e por este motivo é muito difícil encontrar respostas semelhantes em diferentes épocas.

Tabela 2. Valores médios das variáveis relacionadas a características das bagas de seis variedades de uvas sem sementes durante cinco ciclos de produção. Petrolina, PE, 1997-1998(1).

\begin{tabular}{|c|c|c|c|c|c|c|c|}
\hline \multirow[t]{2}{*}{ Variável } & \multirow[t]{2}{*}{ Ciclo } & \multicolumn{6}{|c|}{ Variedade } \\
\hline & & Vênus & Arizul & $\begin{array}{l}\text { Beauty } \\
\text { Seedless }\end{array}$ & $\begin{array}{l}\text { Thompson } \\
\text { Seedless }\end{array}$ & $\begin{array}{l}\text { Marroo } \\
\text { Seedless }\end{array}$ & Canner \\
\hline \multirow{6}{*}{$\begin{array}{l}\text { Comprimento } \\
\text { de bagas (mm) }\end{array}$} & $1^{\circ}$ & $21,53 a$ & $20,13 a$ & $17,73 a$ & $20,47 a b$ & $22,33 a$ & $22,50 \mathrm{a}$ \\
\hline & $2^{\underline{o}}$ & $19,53 \mathrm{ab}$ & $19,90 \mathrm{a}$ & $18,37 \mathrm{a}$ & $22,67 \mathrm{a}$ & 20,50ab & $20,90 \mathrm{ab}$ \\
\hline & $3^{-}$ & $19,43 \mathrm{ab}$ & $17,80 \mathrm{~b}$ & $18,37 \mathrm{a}$ & $19,57 \mathrm{~b}$ & $19,13 b c$ & $20,23 b$ \\
\hline & $4^{o}$ & $19,83 \mathrm{ab}$ & $17,50 \mathrm{~b}$ & $18,17 \mathrm{a}$ & $19,27 b$ & $19,53 b c$ & $20,60 a b$ \\
\hline & $5^{\circ}$ & $18,13 b$ & $18,35 \mathrm{a}$ & $16,93 a$ & $19,59 \mathrm{~b}$ & $17,77 \mathrm{c}$ & $20,77 \mathrm{ab}$ \\
\hline & Média & 16,69 & 18,55 & 17,91 & 20,31 & 19,85 & 21,00 \\
\hline \multirow{6}{*}{$\begin{array}{l}\text { Diâmetro de bagas } \\
(\mathrm{mm})\end{array}$} & $1^{\underline{O}}$ & $19,73 a$ & $17,20 \mathrm{a}$ & $15,40 \mathrm{bc}$ & $16,37 \mathrm{ab}$ & $20,53 a$ & $17,90 \mathrm{a}$ \\
\hline & $2^{\underline{o}}$ & $17,90 \mathrm{~b}$ & $16,70 \mathrm{ab}$ & $15,27 b c$ & $17,70 \mathrm{a}$ & $18,27 \mathrm{~b}$ & $16,70 \mathrm{ab}$ \\
\hline & $3^{\circ}$ & $17,90 \mathrm{~b}$ & $16,07 \mathrm{ab}$ & $17,16 \mathrm{a}$ & $15,63 b$ & $17,97 \mathrm{~b}$ & $15,83 \mathrm{~b}$ \\
\hline & $4^{\circ}$ & $17,60 \mathrm{bc}$ & $15,10 \mathrm{~b}$ & $16,33 \mathrm{ab}$ & $15,23 \mathrm{~b}$ & $18,30 \mathrm{~b}$ & $15,90 \mathrm{~b}$ \\
\hline & $5^{\circ}$ & $16,00 \mathrm{c}$ & $15,54 \mathrm{ab}$ & $14,34 \mathrm{c}$ & $15,15 \mathrm{~b}$ & $16,22 \mathrm{c}$ & $15,74 \mathrm{~b}$ \\
\hline & Média & 17,83 & 16,03 & 15,70 & 16,01 & 18,26 & 16,41 \\
\hline \multirow{6}{*}{$\begin{array}{l}\text { Volume } \\
\text { de } 100 \text { bagas }(\mathrm{mL})\end{array}$} & $1^{\circ}$ & $486,67 \mathrm{a}$ & $313,33 a$ & $214,00 \mathrm{a}$ & $316,67 a$ & $560,00 \mathrm{a}$ & $351,67 \mathrm{a}$ \\
\hline & $2^{\underline{o}}$ & $333,33 b$ & $300,00 \mathrm{ab}$ & $206,67 \mathrm{a}$ & $240,00 \mathrm{~b}$ & $393,33 b$ & $316,67 \mathrm{ab}$ \\
\hline & $3^{\circ}$ & $330,00 \mathrm{~b}$ & $210,00 \mathrm{ab}$ & $261,67 \mathrm{a}$ & $238,33 b$ & $330,00 \mathrm{bc}$ & $270,00 \mathrm{~b}$ \\
\hline & $4^{\circ}$ & $341,67 b$ & $230,00 \mathrm{~b}$ & $253,33 a$ & $230,00 \mathrm{~b}$ & $303,33 c$ & $293,33 \mathrm{ab}$ \\
\hline & $5^{\circ}$ & $280,00 \mathrm{~b}$ & $246,00 \mathrm{~b}$ & $210,00 \mathrm{a}$ & $256,67 \mathrm{ab}$ & $300,00 \mathrm{c}$ & $306,67 \mathrm{ab}$ \\
\hline & Média & 354,33 & 253,85 & 229,13 & 256,33 & 377,33 & 307,67 \\
\hline \multirow[t]{6}{*}{ Peso de bagas (g) } & $1^{\underline{0}}$ & $5,25 \mathrm{a}$ & $3,30 \mathrm{a}$ & $2,29 a$ & $3,35 \mathrm{a}$ & $5,93 a$ & $3,67 \mathrm{a}$ \\
\hline & $2^{\underline{o}}$ & $3,57 \mathrm{~b}$ & $3,66 \mathrm{a}$ & $2,15 \mathrm{a}$ & $2,54 \mathrm{ab}$ & $4,21 \mathrm{~b}$ & $3,27 \mathrm{a}$ \\
\hline & $3^{\circ}$ & $3,52 b$ & $2,25 b$ & $2,77 \mathrm{a}$ & $2,52 \mathrm{~b}$ & $3,52 b c$ & $2,95 \mathrm{a}$ \\
\hline & $4^{\circ}$ & $3,64 b$ & $2,33 b$ & $2,66 \mathrm{a}$ & $2,43 b$ & $3,21 \mathrm{c}$ & $2,99 a$ \\
\hline & $5^{\circ}$ & $2,95 \mathrm{~b}$ & $2,63 \mathrm{ab}$ & $2,19 a$ & $2,73 \mathrm{ab}$ & $3,13 c$ & $3,17 \mathrm{a}$ \\
\hline & Média & 3,79 & 2,71 & 2,41 & 2,71 & 4,00 & 3,21 \\
\hline
\end{tabular}

${ }^{(1)}$ Médias seguidas pela mesma letra na coluna não diferem entre si pelo teste de Tukey $(\mathrm{p}<0,05)$. 
O teor de sólidos solúveis totais variou de $15,52^{\circ}$ Brix na 'Beauty Seedless' a $19,66^{\circ}$ Brix na 'Vênus'. Os valores obtidos nas variedades Vênus, Arizul e Thompson Seedless foram superiores aos exigidos pelas normas de exportação de uva de mesa para os Estados Unidos na classe US Extra (Bleinroth, 1993; Carraro \& Cunha, 1994, citados por Gonçalves et al., 1996). Apenas a variedade Canner apresentou teor de sólidos solúveis totais inferior ao mínimo recomendado de $15^{\circ}$ Brix na região do Vale do Rio São Francisco (Gayet, 1993; Albuquerque, 1996). Quanto à acidez total titulável, Carvalho \& Chitarra (1984) comentam que valores acima de $1,5 \%$ podem ser considerados elevados; entretanto, as médias, em todas as variedades, foram inferiores a 1,0 $\mathrm{g}$ de ácido tartárico por $100 \mathrm{~mL}$ de suco, o que significa que todas elas apresentaram baixa acidez, conseqüência também das elevadas temperaturas da região (Figura 1), que, segundo Calò et al. (1996), favorecem a redução da acidez dos frutos.

Os ciclos de produção ou épocas de poda apresentaram diferenças significativas quanto aos valores observados na relação SST/ATT. Isto é uma con- seqüência da influência exercida pelas alterações climáticas ao longo do ano sobre a acumulação de açúcares e redução da acidez durante a maturação dos frutos. As maiores relações SST/ATT foram observadas no quinto ciclo, em quase todas as variedades, podendo estar relacionadas às elevadas temperaturas médias durante os meses de outubro e novembro de 1998 (Figura 1), que coincidiram com a fase de maturação neste ciclo de produção.

As condições climáticas do Vale do Rio São Francisco, com temperaturas médias elevadas durante a fase de maturação, favorecem maior concentração de açúcares e a redução da acidez nos frutos, resultando em valores de relação SST/ATT iguais ou superiores a 20:1, considerados adequados em uvas de mesa (Gayet, 1993), contribuindo para a melhoria do sabor e qualidade da uva.

O peso dos ramos podados constitui uma medida do vigor vegetativo da planta. As variedades Thompson Seedless e Canner destacaram-se apresentando as maiores médias para esta variável, em todos os ciclos de produção (Tabela 4).

Tabela 3. Valores médios nas variáveis relacionadas à composição química dos frutos de seis variedades de uvas sem sementes durante cinco ciclos de produção. Petrolina, PE, 1997-1998 ${ }^{(1)}$.

\begin{tabular}{|c|c|c|c|c|c|c|c|}
\hline \multirow[t]{2}{*}{ Variável $^{(2)}$} & \multirow[t]{2}{*}{ Ciclo } & \multicolumn{6}{|c|}{ Variedade } \\
\hline & & Vênus & Arizul & $\begin{array}{c}\text { Beauty } \\
\text { Seedless }\end{array}$ & $\begin{array}{c}\text { Thompson } \\
\text { Seedless }\end{array}$ & $\begin{array}{c}\text { Marroo } \\
\text { Seedless }\end{array}$ & Canner \\
\hline \multirow{6}{*}{$\begin{array}{l}\text { Sólidos solúveis } \\
\text { totais (SST) }\end{array}$} & $1^{0}$ & $18,20 \mathrm{ab}$ & $13,90 \mathrm{~b}$ & $14,40 \mathrm{ab}$ & $16,30 \mathrm{~b}$ & $13,80 \mathrm{a}$ & $12,60 \mathrm{~b}$ \\
\hline & $2^{\mathrm{o}}$ & $21,97 \mathrm{a}$ & $17,20 \mathrm{ab}$ & $16,67 \mathrm{a}$ & $16,77 b$ & $15,43 \mathrm{a}$ & $16,50 \mathrm{a}$ \\
\hline & $3^{\circ}$ & $18,13 b$ & $16,10 \mathrm{ab}$ & $13,50 \mathrm{~b}$ & $19,17 \mathrm{ab}$ & $16,50 \mathrm{a}$ & $13,33 b$ \\
\hline & $4^{0}$ & $17,47 \mathrm{~b}$ & $17,67 \mathrm{ab}$ & $16,00 \mathrm{ab}$ & $16,50 \mathrm{~b}$ & $16,97 \mathrm{a}$ & $13,03 \mathrm{~b}$ \\
\hline & $5^{\circ}$ & $21,57 \mathrm{a}$ & $17,93 a$ & 16,30ab & $21,43 a$ & $17,60 \mathrm{a}$ & $14,80 \mathrm{ab}$ \\
\hline & Média & 19,66 & 16,93 & 15,52 & 18,30 & 16,41 & 14,05 \\
\hline \multirow{6}{*}{$\begin{array}{l}\text { Acidez total } \\
\text { titulável (ATT) }\end{array}$} & $1^{\mathrm{o}}$ & $0,78 \mathrm{abc}$ & $1,04 \mathrm{ab}$ & $1,26 \mathrm{a}$ & $1,38 \mathrm{a}$ & $0,62 \mathrm{ab}$ & $0,81 \mathrm{a}$ \\
\hline & $2^{\mathrm{o}}$ & $0,71 b$ & $0,78 b c$ & $0,64 c$ & $0,66 \mathrm{c}$ & $0,71 \mathrm{a}$ & $0,60 \mathrm{ab}$ \\
\hline & $3^{\mathrm{o}}$ & $0,84 \mathrm{a}$ & $1,15 \mathrm{a}$ & $0,86 b$ & $0,91 b$ & $0,75 a$ & $0,47 b$ \\
\hline & $4^{\mathrm{o}}$ & $0,78 \mathrm{ab}$ & $0,96 \mathrm{ab}$ & $1,17 \mathrm{a}$ & $0,75 b c$ & $0,70 \mathrm{a}$ & $0,64 \mathrm{ab}$ \\
\hline & $5^{\circ}$ & $0,53 c$ & $0,63 \mathrm{c}$ & $0,72 b c$ & $0,77 \mathrm{bc}$ & $0,50 \mathrm{~b}$ & $0,64 \mathrm{ab}$ \\
\hline & Média & 0,72 & 0,91 & 0,88 & 0,82 & 0,66 & 0,61 \\
\hline Relação & $1^{\mathrm{O}}$ & $23,30 \mathrm{bc}$ & $13,40 \mathrm{~b}$ & $11,40 \mathrm{~b}$ & $11,80 \mathrm{~b}$ & $22,40 \mathrm{~b}$ & $15,60 \mathrm{~b}$ \\
\hline \multirow[t]{5}{*}{ SST/ATT } & $2^{\mathrm{o}}$ & $31,15 b$ & $22,50 \mathrm{ab}$ & $26,37 \mathrm{a}$ & $26,00 \mathrm{a}$ & $21,77 b$ & $27,77 \mathrm{a}$ \\
\hline & $3^{\mathrm{o}}$ & $21,73 \mathrm{c}$ & $14,18 b$ & $15,74 b$ & $21,29 \mathrm{a}$ & $22,13 b$ & $29,00 \mathrm{a}$ \\
\hline & $4^{\mathrm{o}}$ & $22,39 \mathrm{c}$ & $18,49 \mathrm{~b}$ & $13,80 \mathrm{~b}$ & $22,13 a$ & $24,48 b$ & $20,38 b$ \\
\hline & $5^{\circ}$ & $40,78 \mathrm{a}$ & $28,70 \mathrm{a}$ & $22,87 \mathrm{a}$ & $27,79 \mathrm{a}$ & $34,83 \mathrm{a}$ & $23,04 \mathrm{ab}$ \\
\hline & Média & 28,57 & 20,00 & 19,05 & 23,34 & 25,54 & 24,32 \\
\hline
\end{tabular}

${ }^{(1)}$ Médias seguidas pela mesma letra na coluna não diferem entre si pelo teste de Tukey $(\mathrm{p}<0,05) .{ }^{(2)} \mathrm{SST}$ : medida em ${ }^{\circ}$ Brix; ATT: medida em g de ácido tartárico por $100 \mathrm{~mL}$ de suco. 
Tabela 4. Valores médios das variáveis relacionadas a características de vigor e produção das plantas de seis variedades de uvas sem sementes em cinco ciclos de produção. Petrolina, PE, 1997-1998(1).

\begin{tabular}{|c|c|c|c|c|c|c|c|}
\hline \multirow[t]{3}{*}{ Variável } & \multirow[t]{3}{*}{ Ciclo } & \multicolumn{6}{|c|}{ Variedade } \\
\hline & & Vênus & Arizul & Beauty & Thompson & Marroo & Canner \\
\hline & & & & Seedless & Seedless & Seedless & \\
\hline \multirow{6}{*}{$\begin{array}{l}\text { Peso dos ramos } \\
(\mathrm{kg})\end{array}$} & $1^{\mathrm{O}}$ & $1,65 \mathrm{Ca}$ & $3,65 \mathrm{BCa}$ & $3,23 \mathrm{BCa}$ & $9,51 \mathrm{Aa}$ & $4,26 \mathrm{Bab}$ & $5,70 \mathrm{Ba}$ \\
\hline & $2^{\underline{o}}$ & $0,76 \mathrm{Da}$ & $2,58 \mathrm{CDa}$ & $2,58 \mathrm{CDa}$ & $8,68 \mathrm{Aa}$ & $5,02 \mathrm{BCa}$ & $6,28 \mathrm{ABa}$ \\
\hline & $3^{-}$ & - & - & - & - & - & - \\
\hline & $4^{\mathrm{o}}$ & $1,02 \mathrm{Ba}$ & $2,52 \mathrm{Ba}$ & $2,14 \mathrm{Ba}$ & 9,76Aa & 2,57Bab & $5,63 \mathrm{Aa}$ \\
\hline & $5^{\circ}$ & $0,73 \mathrm{Ba}$ & $1,42 \mathrm{Ba}$ & $1,17 \mathrm{Ba}$ & $4,11 \mathrm{Ab}$ & $1,75 \mathrm{ABa}$ & $2,67 \mathrm{ABb}$ \\
\hline & Média & 1,04 & 2,55 & 2,28 & 8,02 & 3,38 & 5,05 \\
\hline \multirow{6}{*}{$\begin{array}{l}\text { Produção/planta } \\
\text { (kg) }\end{array}$} & $1^{\mathrm{O}}$ & $6,36 \mathrm{Bb}$ & $12,38 \mathrm{Aa}$ & $11,41 \mathrm{Aa}$ & $3,97 \mathrm{Ba}$ & $13,43 \mathrm{Aa}$ & $4,46 \mathrm{Ba}$ \\
\hline & $2^{\underline{o}}$ & $12,55 \mathrm{Aa}$ & $1,70 \mathrm{Bc}$ & $2,17 \mathrm{Bb}$ & $1,35 \mathrm{Ba}$ & $5,43 \mathrm{Bb}$ & $1,71 \mathrm{Ba}$ \\
\hline & $3^{-}$ & $7,22 \mathrm{Ab}$ & $5,84 \mathrm{Abc}$ & $4,23 \mathrm{ABb}$ & $1,14 \mathrm{Ba}$ & 7,39Ab & $1,27 \mathrm{Ba}$ \\
\hline & $4^{\circ}$ & 7,87Ab & 4,51ABbc & $3,67 \mathrm{Bb}$ & $1,86 \mathrm{Ba}$ & 7,83Ab & $1,89 \mathrm{Ba}$ \\
\hline & $5^{\circ}$ & $13,20 \mathrm{Aa}$ & $6,47 \mathrm{BCb}$ & 9,49ABa & $3,80 \mathrm{Ca}$ & $5,27 \mathrm{Cb}$ & $4,39 \mathrm{Ca}$ \\
\hline & Média & 9,45 & 6,30 & 6,46 & 2,48 & 7,82 & 2,78 \\
\hline \multirow{6}{*}{$\begin{array}{l}\text { Número de } \\
\text { cachos/planta }\end{array}$} & $1^{\underline{O}}$ & $40,00 \mathrm{BCb}$ & $49,00 \mathrm{Ba}$ & $81,00 \mathrm{Aa}$ & $28,00 \mathrm{BCa}$ & $77,00 \mathrm{Aa}$ & $25,00 \mathrm{Ca}$ \\
\hline & $2^{\mathrm{o}}$ & $63,00 \mathrm{Aa}$ & $7,00 \mathrm{BCc}$ & $10,00 \mathrm{BCc}$ & $4,00 \mathrm{Cb}$ & $27,00 \mathrm{Bb}$ & $5,00 \mathrm{Ca}$ \\
\hline & $3^{-}$ & $62,00 \mathrm{Aa}$ & $34,00 \mathrm{Bab}$ & $28,00 \mathrm{BCbc}$ & $9,00 \mathrm{Cab}$ & $63,00 \mathrm{Aa}$ & $8,00 \mathrm{Ca}$ \\
\hline & $4^{\circ}$ & $63,00 \mathrm{Aa}$ & $20,00 \mathrm{BCbc}$ & $21,00 \mathrm{BCbc}$ & $11,00 \mathrm{Cab}$ & $36,00 \mathrm{Bb}$ & $11,00 \mathrm{Ca}$ \\
\hline & $5^{-}$ & 76,00Aa & $28,00 \mathrm{BCbc}$ & $36,00 \mathrm{Bb}$ & $20,00 \mathrm{BCab}$ & $39,00 \mathrm{Bb}$ & $15,00 \mathrm{Ca}$ \\
\hline & Média & 61,00 & 28,00 & 37,00 & 15,00 & 48,00 & 13,00 \\
\hline
\end{tabular}

${ }^{(1)}$ Médias seguidas pela mesma letra, maiúscula na linha e minúscula na coluna, não diferem entre si pelo teste de Tukey $(\mathrm{p}<0,05)$.

As variedades e os ciclos de produção avaliados apresentaram diferenças significativas em relação às variáveis de produção e número de cachos por planta. 'Vênus', 'Marroo Seedless', 'Arizul' e 'Beauty Seedless' alternaram ciclos de maior produção com outros de produção reduzida. 'Thompson Seedless' e 'Canner' apresentaram produção e número de cachos por planta mais constantes ao longo do tempo (Tabela 4). No entanto estas duas variedades apresentaram um aumento na produção, embora não significativo, do quarto para o quinto ciclo e no mesmo período suas plantas apresentaram redução no peso dos ramos, comprovando aquilo que comentam Winkler (1965) e Hidalgo (1999), quanto à relação inversa entre vigor e produção.

As produções médias, em t/ha/ano, correspondem a 24, 20, 16, 16, 11 e 6 para 'Vênus', 'Marroo Seedless', 'Arizul', 'Beauty Seedless', 'Canner' e 'Thompson Seedless'. Pode-se considerar que as produtividades alcançadas pelas variedades Vênus e Marroo Seedless são satisfatórias, demonstrando, portanto, potencial para cultivo no Submédio São Francisco.

\section{Conclusões}

1. Entre as variedades estudadas, apenas 'Vênus' produz cachos com tamanho característico dos padrões varietais.

2. 'Vênus' e 'Marroo Seedless' apresentam bagas que atendem às exigências para exportação.

3. O teor de sólidos solúveis estão acima dos valores mínimos exigidos para comercialização de uvas de mesa.

4. O vigor excessivo mostra ser uma característica que inviabiliza a produção comercial das variedades Thompson Seedless e Canner.

5. As produtividades encontradas nas variedades Vênus e Marroo Seedless demonstram que elas possuem potencial para cultivo nas condições tropicais do Submédio São Francisco.

\section{Referências}

ALBUQUERQUE, T. C. S. de. Uvas para exportação aspectos técnicos da produção. Brasília : Embrapa-SPI, 1996. 53 p. (Série Publicações Técnicas FRUPEX, 25). 
BLEINROTH, E. W. Normas para seleção e classificação da uva. In: GORGATTI NETTO, A.; GAYET, J. P.; BLEINROTH, E. F. G.; MATALLO, M.; GARCIA, E.; GARCIA, A. E.; ARDITO, E. F. G.; BORDIN, M. Uvas para exportação: procedimentos de colheita e pós-colheita. Brasília : Embrapa-SPI, 1993. 40 p. (Série Publicações Técnicas FRUPEX, 2).

CALÒ, A.; LIUNI, C. S.; COSTACURTA, A.; COLAPIETRA, M.; RENNA, D. Le uve da tavola. Conegliano : Istituto Sperimentale per la Viticoltura, 1989. $319 \mathrm{p}$.

CALÒ, A.; TOMASI, D.; CRESPAN, M.; COSTACURTA, A. Relationship between environmental factors and the dynamics of growth of the grapevine. Acta Horticulturae, Leuven, v. 427, p. 217-231, 1996.

CAMARGO, U. A.; MASHIMA, C. H.; CZERMAINSKI, A. B. C. Avaliação de cultivares de uvas apirênicas no Vale do São Francisco. Bento Gonçalves : Embrapa-CNPUV, 1997. 8 p. (Comunicado Técnico, 26).

CARVALHO, V. D.; CHITARRA, M. I. F. Aspectos qualitativos da uva. Informe Agropecuário, Belo Horizonte, v. 10, n. 117 , p. $75-79,1984$.

FERRI, C. P. Caracterização agronômica e fenológica de cultivares e clones de videira (Vitis spp.) mantidos no Instituto Agronômico, Campinas, SP. Piracicaba : ESALQ, 1994. 89 p. Dissertação de Mestrado.

GAYET, J. P. Características das frutas de exportação. In: GORGATTI NETTO, A.; GAYET, J. P.; BLEINROTH, E. F. G.; MATALLO, M.; GARCIA, E.; GARCIA, A. E.; ARDITO, E. F. G.; BORDIN, M. Uvas para exportação: procedimentos de colheita e pós-colheita. Brasília : Embrapa-SPI, 1993. 40 p. (Série Publicações Técnicas FRUPEX, 2).

GONÇALVES, J. S.; AMARO, A. A.; MAIA, M. L.; SOUZA, S. A. M. Estrutura de produção e de mercado da uva de mesa brasileira. Agricultura em São Paulo, São Paulo, v. 43, n. 1, p. 43-93, 1996.

HALE, C. R.; BUTTROSE, M. Effect of temperature on ontogeny of berries of Vitis vinifera L. cv. Cabernet Sauvignon. Journal of the American Society for Horticultural Science, Alexandria, v. 99, n. 5, p. 390394, 1974.

HIDAlgO, L. Poda de la vid. 5. ed. Madrid : Mundi, 1999. $259 \mathrm{p}$.

LEÃO, P. C. de S.; JÚNIOR LINO, E. da C.; SANTOS, E. da S. Efeitos do CPPU e ácido giberélico sobre o tamanho de bagas da uva Perlette cultivada no Vale do São Francisco. Revista Brasileira de Fruticultura, Cruz das Almas, v. 21, n. 1, p. 74-78, 1999.

MATHEOU, A.; STAVROPOULOS, N.; SAMARAS, S. Studies on table grape germoplasm growth in Northern Greece: II. Seedlessness, berry and must characteristics. Vitis, Siebeldingen, v. 34, n. 4, p. 217-220, 1995.

MOORE, J. N. ; BROWN, E. 'Venus' grape. HortScience, Alexandria, v. 12, n. 6, p. 585, 1977.

SCHUCK, E. Efeitos de reguladores de crescimento sobre o peso dos cachos, bagas e maturação da uva de mesa, cv. 'Vênus'. Revista Brasileira de Fruticultura, Cruz das Almas, v. 16, n. 1, p. 295-306, 1994.

SILVA, P. C. G. da; SOUZA LEÃO, P. C. de; CERDAN, C.; DENIS, S.; CHOUDHURY, M. M.; BENTZEN, M. da C. P.; BARRETO, M. C. A cadeia produtiva da uva de mesa do Nordeste do Brasil. In: CASTRO, A. M. G. de; LIMA, S. M. V.; GOEDERT, W. J.; FREITAS FILHO, A. de; VASCONCELOS, J. R. P. (Ed.). Cadeias produtivas e sistemas naturais: prospecção tecnológica. Brasília : Embrapa-SPI, 1998. p. 529-564.

WAGNER, R.; TRUEL, P. Nouvelles variétés de raisins de table et de raisins secs. Paris : Station de Viticulture de Montpellier, 1988. p. 242.

WINKLER, A. J. Viticultura. México : Editorial Continental, 1965. $792 \mathrm{p}$. 